Ann. Biol. anim. Bioch. Biophys., I967, 7 (3), 245-259.

\title{
CYCLE SAISONNIER DE REPRODUCTON DU CAMPAGNOL DES GHAMPS MICROTUS ARVALIS
}

\author{
Lise MARTINET \\ a vec la collaboration technique de Monique Meunier et P. Bureau \\ Station centrale de Physiologie animale, \\ Centre national de Recherches zootechniques, 78 - Jouy-en-Josas \\ Institut national de la Recherche agronomique
}

\section{SOMMAIRE}

Le travail a porté sur 5 années. Des piégeages mensuels ont été effectués. Les techniques utilisées ont permis de connaître la croissance des animaux, leur âge d'après le poids du cristallin, leur état de maturation sexuelle, leur fertilité.

Il existe deux populations successives au cours de l'année, la première composée d'individus naissant de mars à juin, à croissance rapide et à durée de vie courte, la seconde composée d'individus naissant à partir de juillet à croissance lente et durée de vie longue ; c'est cette deuxième population qui passe l'hiver et donne les premières portées de l'année suivante.

Le cycle annuel de reproduction a été observé ; comme la durée de ce cycle n'est pas constante d'une année à l'autre, le photopériodisme n'en est pas le seul facteur responsable.

\section{INTRODUCTION}

CHAPPELIER (I937) observe chez Microtus arvalis une activité génitale du printemps à l'automne avec repos hivernal. DELOST (I955) reprenant ces observations, trouve en effet, que Microtus arvalis a une saison sexuelle très nette, bien délimitée de mars à octobre et constate une atrophie complète des organes génitaux du mâle et de la femelle pendant le repos hivernal. Cependant, STEIN (I953) remarque des variations de la durée de la saison de reproduction de ce rongeur suivant les biotopes. Maximov (I948) observe une bonne reproduction en hiver sous les meules de paille. SPITz (I963) travaillant en Vendée, sur les lieux oì cette étude a été menée, constate que la saison de reproduction s'étend de février à octobre avec parfois des arrêts précoces de la reproduction (en juin I959 et en juin-juillet I96I). 
Ces variations dans le cycle annuel de reproduction se retrouvent chez d'autres espèces de Microtus.

Si Microtus agrestis présente un arrêt hivernal de la reproduction bien net en Angleterre (BAKER et RANSON, I932), dans le Tarn, RAYNAUD (I95I) observe des gestations hivernales.

Aux Etats-Unis, chez Microtus pennsylvanicus (BEER et MAC LEOD, I96I) et chez Microtus oregoni (CoWAN et ARSENAULT, I954), on observe une certaine reproduction en hiver. Microtus californicus (GREENWALD, I957) se reproduit toute l'année avec deux maxima en mars-avril et en septembre-octobre.

Cette étude a été poursuivie pendant 5 ans en Vendée (latitude $46^{\circ} 3^{\prime}$ ) dans le marais de Luçon pour étudier le cycle annuel de reproduction du Campagnol des champs (Microtus arvalis).

\section{MATÉRIEL, E'T MÉTHODE}

Les piégeages ont été effectués mensuellement sur les digues, dans les luzernes des polders côtiers ainsi que dans les prés salés de la zone maritime, au fond de la baie de l'Aiguillon, en Vendée.

De I962 à I965, deux milieux ont été étudiés : les prés salés à végétation halophile dense et les digues couvertes de végétation herbacée. En I966, l'accès des digues étant impossible à cause de l'installation de parcs à moutons, les études ont été effectuées dans les prés salés et les luzernes du polder.

Dans le tableau I, ont été réunies les dates de piégeages et le nombre d'animaux capturés au cours des 5 années. En 1962 et $196_{3}$, les densités de population étaient très faibles ; certains mois, aucune capture n'a été réalisée.

Les animaux ont été pris vivants grâce à un système de pièges-dortoirs qui les protègent de la chaleur ou du froid excessif et leur permettent de s'alimenter. Les animaux sont tués à l'éther dès leur retour au laboratoire.

\section{Croissance des animaux}

Ils sont pesés et mesurés (longueur tête-corps) juste après la mort.

\section{Détermination de l'âge}

Elle a été faite par la technique de la pesée du cristallin, mise au point par LORD (I959); une courbe standard a été établie sur des animaux d'élevage (MARTINET, 1966a).

\section{La ferititié}

Chez les mâles. - Les testicules ont été pesés et fixés au Bouin Hollande. Ceux des animaux de 2 à 4 mois ont été coupés à 7 microns, colorés à l'acide periodique Schiff hématoxyline.

Une étude quantitative de la spermatogenèse a été faite suivant la méthode utilisée dans un précédent travail (MARTINET, I963).

Les épididymes ont été congelés immédiatement après la mort, dans une solution de saccharose à o,25 mol pour permettre une étude ultérieure des réserves spermatiques épididymaires par broyage suivant la technique de DOUNCE (1943).

Les mâles ont été considérés en activité sexuelle lorsqu'ils possédaient des réserves spermatiques épididymaires.

Chez les femelles. - Les ovaires et les oviductes ont été prélevés, fixés au Bouin Hollande; le dénombrement des corps jaunes actifs ou en régression dans l'ovaire et des oufs fécondés ou non dans l'oviducte, a été effectué sur des coupes sériées, colorées par le Trichrome de Masson. 


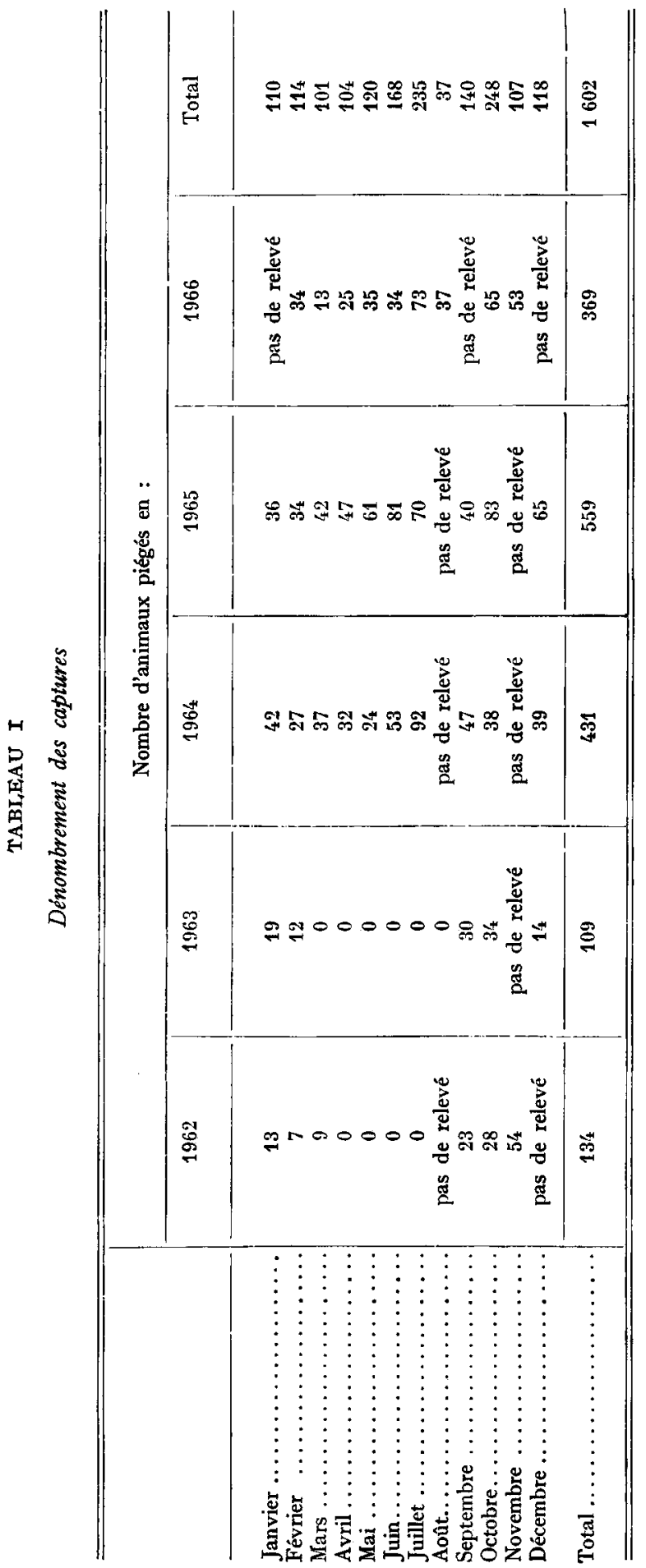


Les sites d'implantation, les foetus ou les cicatrices placentaires sont dénombrés. Les battements du cœur ont été pris comme critère de viabilité des fœetus (le cœur commence à battre le douzième jour de la gestation).

La présence d'une lactation a été retenue d'après l'aspect de la glande mammaire et des trayons.

Les femelles ont été considérées en activité sexuelle lorsqu'il y avait présence de gestation ou de lactation,

\section{RÉSULTATS}

A partir des renseignements rassemblés, nous avons pu étudier d'une part la croissance des animaux, leur durée de vie, la vitesse de leur maturation sexuelle, d'autre part la fécondité de la population au cours de l'année, à savoir : la durée de la saison sexuelle, le nombre d'individus participant à la reproduction et la fertilité de ces individus.

\section{Croissance corporelle des Campagnols}

Dans la figure I pour les mâles, 2 pour les femelles, sont consignés les résultats sur la croissance des Campagnols en fonction de leur date de naissance.

Trois groupes ont été considérés : les Campagnols de $x$ à 2 mois, de 2 à 4 mois, et ceux de 4 à 6 mois. La détermination de l'âge à partir du poids du cristallin a été faite au cours des années I964, I965 et r966.

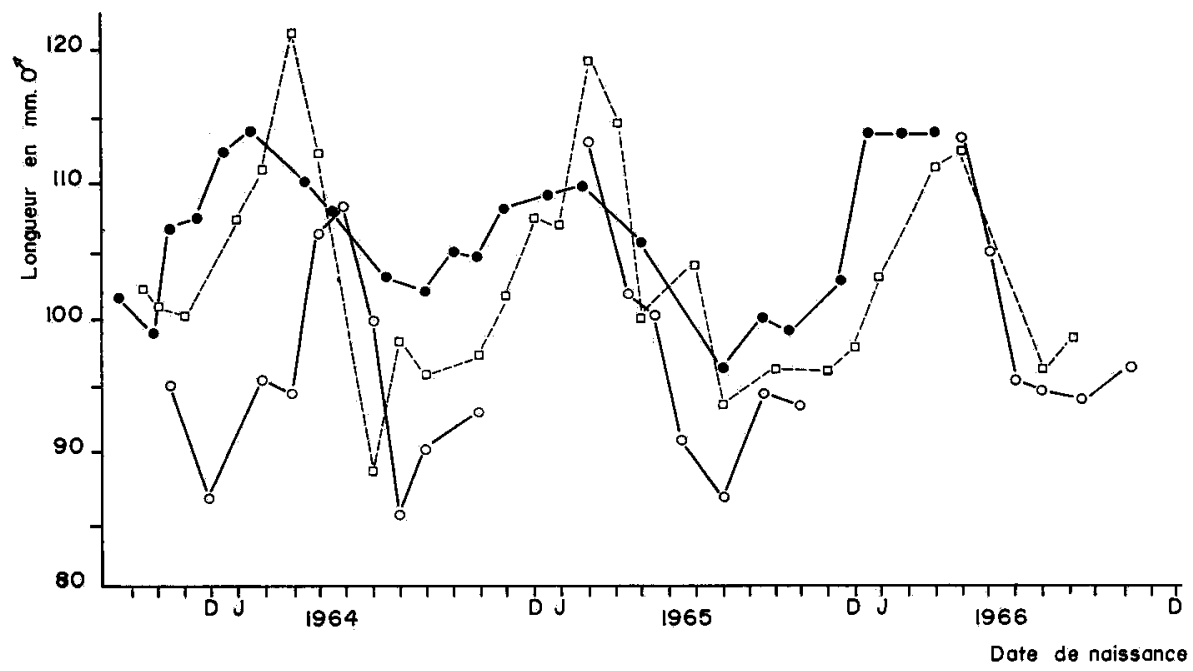

FIG. x. - Croissance des Campagnols mâles en fonction de leur date de naissance

La croissance a été mesurée pour les mâles âgés de :

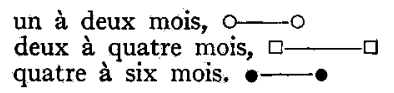

Il existe une différence dans la croissance des animaux en fonction de leur date de naissance. Les animaux nés de janvier-février à avril ont une croissance rapide, au contraire, ceux nés en juillet-août, une croissance lente. 
On peut remarquer dans la figure I, que chez les mâles de I à 2 mois, la croissance maximum a lieu chez les animaux nés en mai I964, février I965, avril I 966 ; la croissance minimum pour ceux nés en juillet I964, juillet I965 et août I965. Chez les mâles de 2 à 4 mois, la croissance maximum a lieu chez les animaux nés en mars I964, février I965, avril I966 et la croissance minimum pour les animaux nés en juin I964, juillet I965, juillet I966. Chez les mâles de 4 à 6 mois, bien que la courbe de

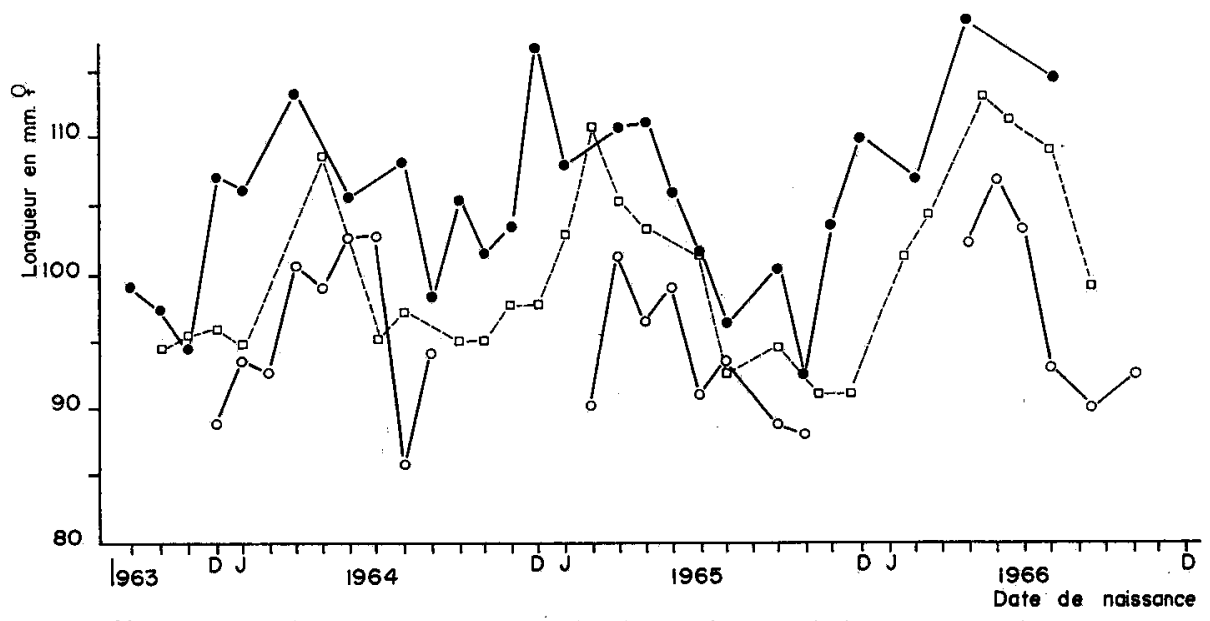

FrG. 2. - Croissance des Campagnols temelles en fonction de leur date de naissance

La croissance a été mesurée pour les femelles âgées de :

un à deux mois; $-\circ$
deux à quatre mois $\square-$
quatre à six mois.

variation de la vitesse de croissance soit plus aplatie, on retrouve des maxima de croissance pour les animaux nés de février à avril, des minima de croissance pour ceux nés de juin à août. En I966, trop peu de mâles de 4 à 6 mois ont été piégés après le mois de mars pour estimer la vitesse de croissance.

Chez les femelles, on trouve des courbes identiques indiquant des croissances différentes suivant la date de naissance.

\section{Durée de vie en fonction de la saison (fig. 3)}

Les animaux nés de février à mai, qui ont une croissance très rapide, semblent mourir très rapidement. Au contraire, les animaux nés à partir de juin ont une croissance beaucoup plus lente et une durée de vie plus longue. Ce serait ces derniers qui passeraient 1'hiver continuant une croissance progressive mais très lente et qui.donneraient les premières portées de l'année suivante.

En effet, on remarque sur la figure 3 qu'en juin-juillet, c'est-à-dire 2 à 4 mois après le début de la reproduction on trouve peu de Campagnols dans la classe 2 à 4 mois, mais essentiellement des individus de o à 2 mois. Au contraire, à partir des mois d'août et septembre, la classe d'âge 2 à 4 mois représente un fort pourcentage de l'ensemble de la population. La plupart des animaux nés de février à mai meurent dans les deux premiers mois de leur vie après avoir donné des portées à croissance plus lente. 
D'octobre à mars, on observe un vieillissement normal de la population, puisqu'il n'y a plus de naissance.
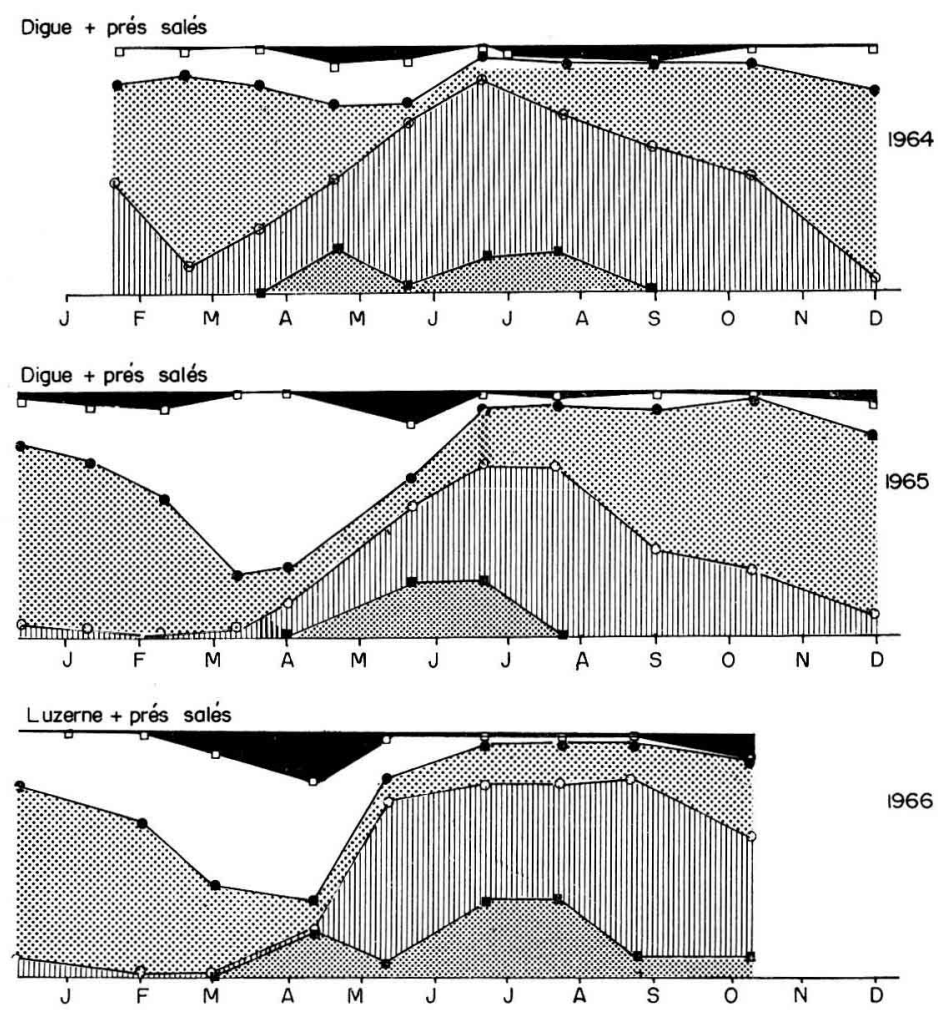

FIG. 3. - Struclure d'âge de la population en fonction de la saison

Les résultats sont exprimés en pourcentage de la population totale:

animaux de moins d'un mois animaux de un à deux mois animaux de deux à quatre mois animaux de quatre à six mois animaux de plus de six mois

3. Age à la maturité sexuelle (fig. 4)

D'après la bibliographie et nos observations personnelles, on sait que chez $\mathrm{Mi}$ crotus arvalis, le mâle est pubère vers le $35^{\mathrm{e}}$ jour, la puberté étant définie par l'apparition de spermatozoïdes dans l'épididyme; la femelle pourrait s'accoupler dès le $2 \mathrm{I}^{\mathrm{e}}$ jour. Nous avons obtenu au laboratoire des ovulations et des fécondations quelquefois suivies de gestation, chez des femelles accouplées au $2 \mathrm{I}^{\mathrm{e}}$ jour.

Dans la figure 4 nous avons représenté le pourcentage de mâles ou de femelles en activité sexuelle au cours de l'année pour trois classes d'âge: 1 à 2 mois, 2 à 4 mois et 4 à 6 mois.

Le pourcentage d'individus en activité sexuelle varie avec les mois de l'année. 
Les mâles de I à 2 mois piégés en avril, mai, juin pour I964, en avril pour I965, en mai pour I966, c'est-à-dire nés en février, mars, avril, sont tous en activité sexuelle. Au contraire, chez ceux piégés de juin à septembre, c'est-à-dire nés de mai à août, il y a une diminution du nombre de mâles actifs. Chez les mâles de I à 2 mois piégés de septembre à décembre, c'est-à-dire nés d'août à novembre, il n'y a plus aucune activité sexuelle.
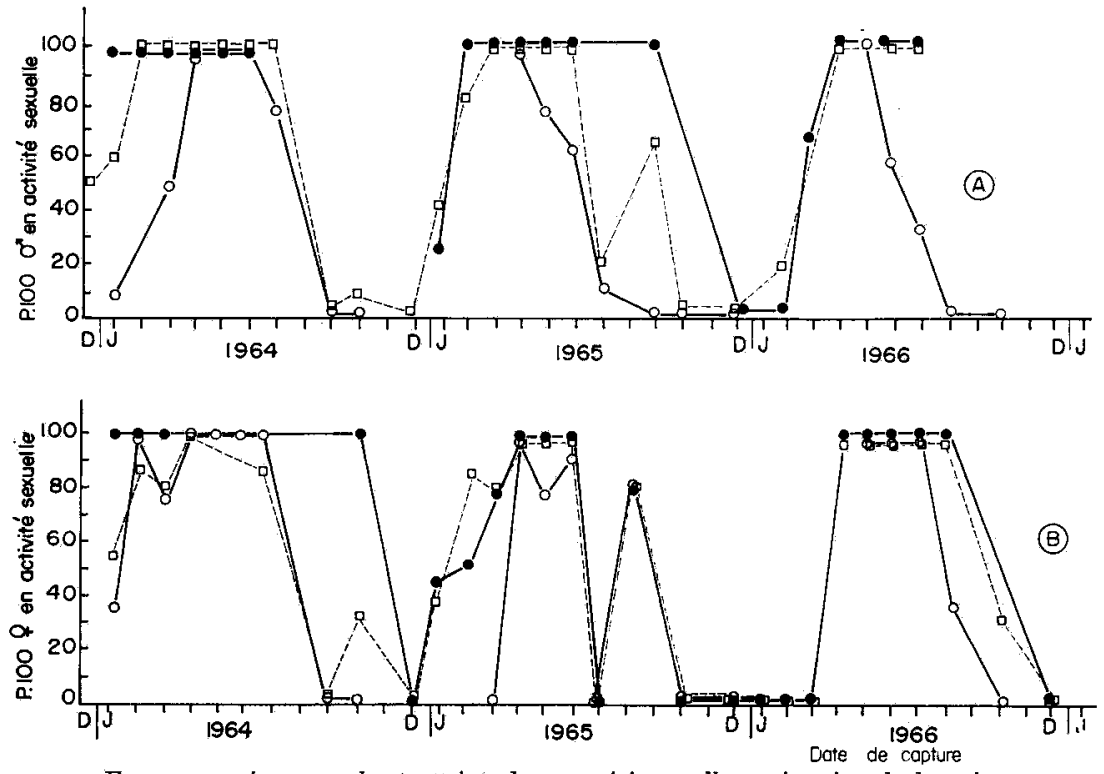

FIG. 4. - Age auquel est atteinte la maturité sexuelle en fonction de la saison

A - Chez les mâles

B $\rightarrow$ chez les femelles

animaux de un à deux mois o- 0

animaux de deux à quatre mois $\square-\square$

animaux de quatre à six mois $\longrightarrow$

Si l'on considère les mâles de 2 à 4 mois, on retrouve le même phénomène ; les animaux nés à partir d'août c'est-à-dire piégés en octobre-novembre, ne sont pas actifs.

A partir d'octobre, ne sont actifs que les mâles de plus de 4 mois, c'est-à-dire nés avant juillet. En décembre, les quelques mâles piégés qui sont actifs sont très âgés (classe d’âge supérieur à 6 mois).

Chez les femelles on observe le même phénomène : les animaux nés à partir de juin-juillet n'atteignent pas la maturité sexuelle l'année de leur naissance.

\section{Durée de la saison de reproduction (fig. 5)}

Pour les 5 années étudiées, la reproduction commence entre le 15 janvier et le I5 février :

en I962: mi-février avec I3 p. Ioo d'individus actifs, en I963: mi-janvier avec $26 \mathrm{p}$. Ioo d'individus actifs, en I964: fin janvier avec 27 p. Ioo d'individus actifs, en I965: mi-janvier avec $36 \mathrm{p}$. Ioo d'individus actifs, en I966: début février avec Io p. Ioo d'individus actifs. 
D'avril à juin, 85 p. Ioo des individus de la population sont fertiles; les quelques animaux ne participant pas à la reproduction étant ceux de moins d'un mois, n'ayant pas encore atteint la maturité sexuelle. A partir de juillet, le pourcentage d'individus sexuellement actifs diminue.

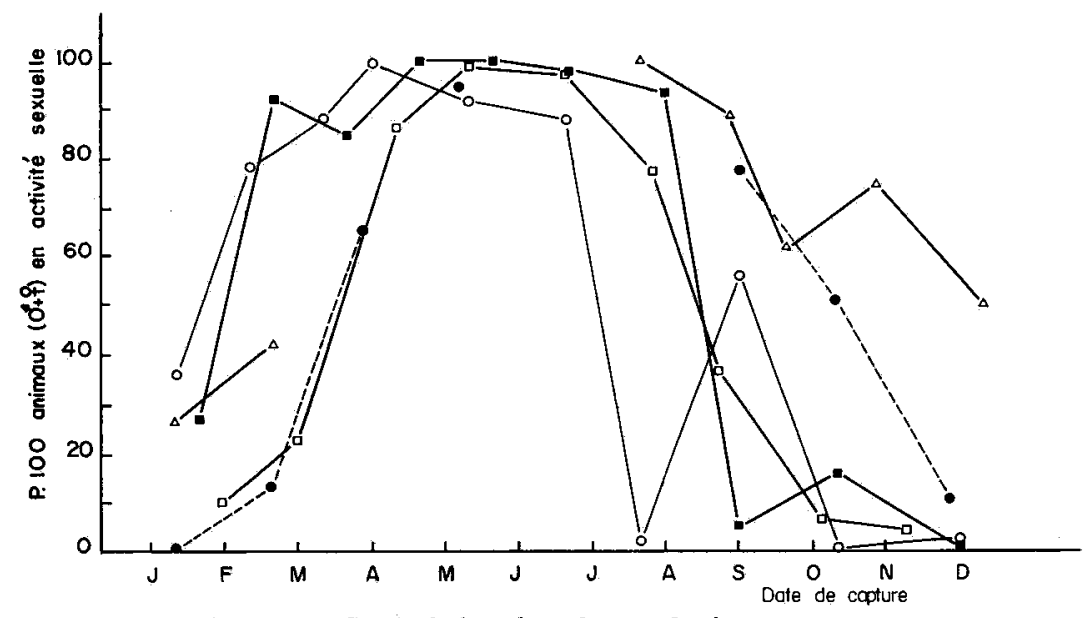

FIG. $5 \cdot$ - Durée de la saison de reproduction et pourcentage

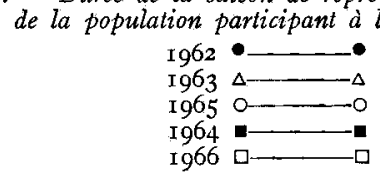

Les dates d'arrêt de reproduction sont beaucoup plus variables : en I964 et I966, le pourcentage d'individus actifs diminue à partir de juillet, ceci correspond à l'apparition d'une population de jeunes Campagnols à croissance lente qui n'atteignent pas la maturité sexuelle avant 1'année suivante. En I962 et I963, la diminution de la reproduction commence deux mois plus tard, à partir de septembre. En I965, on assiste à un arrêt brutal de la reproduction chez tous les individus de la population en juillet, avec une légère reprise en septembre.

On peut noter que certaines années : hiver I963-I964 notamment, il n'y a pas d'arrêt complet de la reproduction. En décembre I963, on trouve $50 \mathrm{p}$. roo et en janvier $1964,27 \mathrm{p}$. Ioo d'individus actifs.

Il n'existe pas de différence dans la durée de la saison sexuelle ni en fonction du sexe, ni en fonction du milieu végétal.

\section{Fertilité des males}

Le poids des testicules et vésicules séminales commence à augmenter en janvierfévrier, passe par un maximum d'avril à juin, puis diminute à partir de juillet. Ceci est valable pour l'ensemble de la population, mais aussi pour les différentes classes d'âge (fig. 6 et 7 ).

Les comptages des différentes catégories cellulaires ont été faits au stade I (ORTAVanT, I958) du cycle de l'épithélium séminifère, caractérisé par la présence de spermatogonies $\mathrm{A}$, de spermatocytes primaires au stade leptotène, au stade pachy- 
tène et de spermatides rondes. Ils permettent de mettre en évidence un rendement différent suivant les mois (tabl. 2).

Ainsi, le nombre de spermatocytes obtenus à partir d'une spermatogonie $\mathrm{A}$, est maximum en avril-mai, le nombre de spermatides obtenues à partir d'un spermatocyte est plus élevé d'avril à septembre que de janvier à mars.

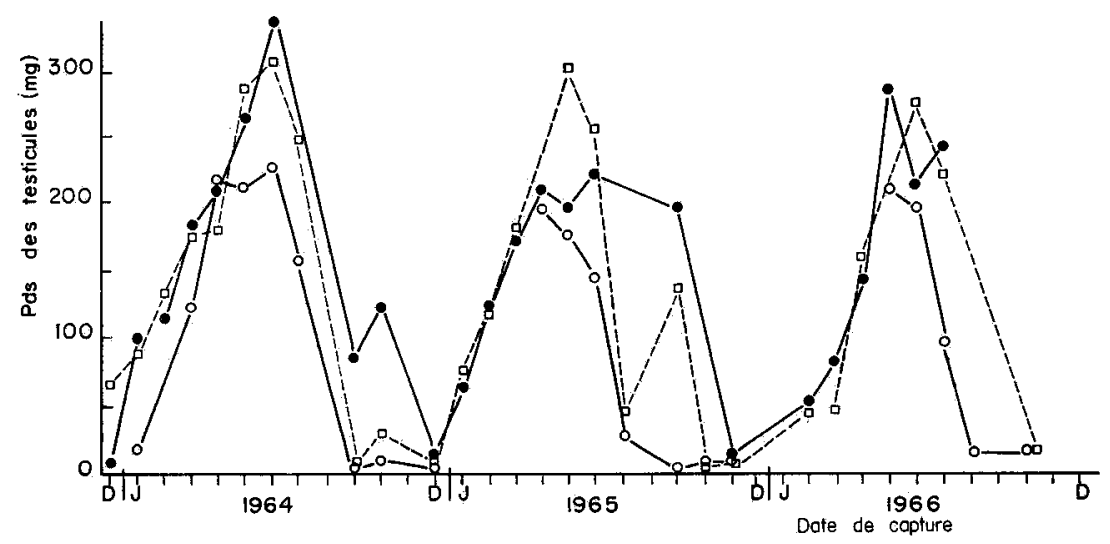

Fig. 6. - Variation du poids des testicules en fonction du cycle anmuel

mâles de un à deux mois o- 0

mâles de deux à quatre mois

mâles de quatre à six mois

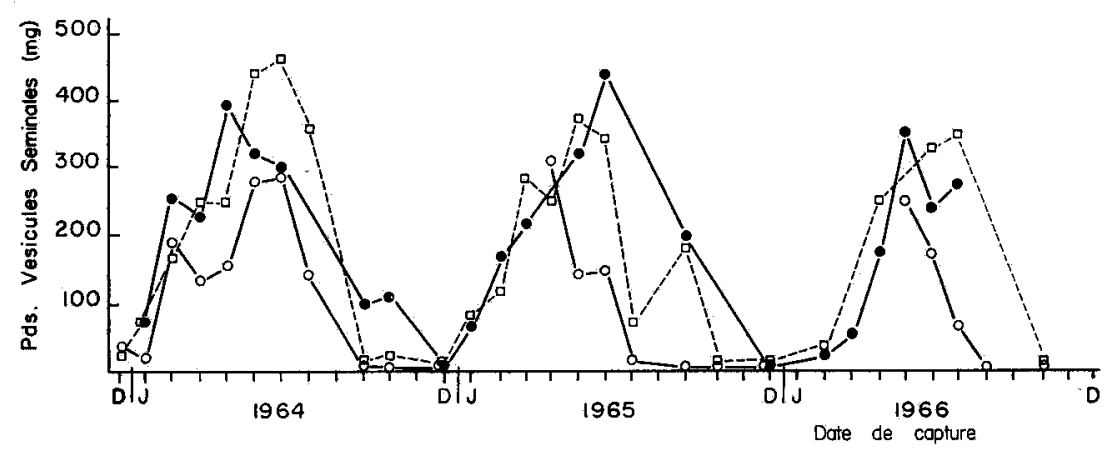

Fig. 7. - Variation du poids dés vésicules séminales en fonction du cycle annuel mâles de un à deux mois
mâles de deux à quatre mois $\square-\square$
mâles de quatre à six mois

\section{Fertilité des femelles}

La fertilité des femelles a été estimée en étudiant d'une part, le nombre d'ovulations, d'autre part, le nombre de foetus et la mortalité embryonnaire.

Le nombre d'ovulations varie avec la saison (fig. $8 \mathrm{~A}$ ).

Il passe de trois corps jaunes par femelle en janvier, à 7 ou 8 en mai-juin, puis diminue à trois en novembre-décembre. 


\begin{tabular}{|c|c|c|c|}
\hline & & 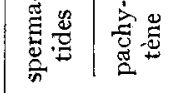 & 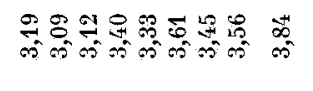 \\
\hline & 苞 & 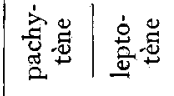 & 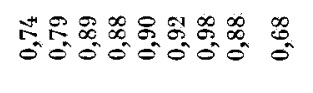 \\
\hline & & 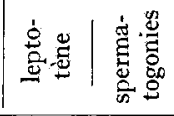 & 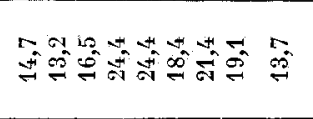 \\
\hline 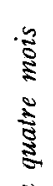 & & 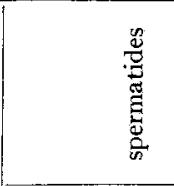 & 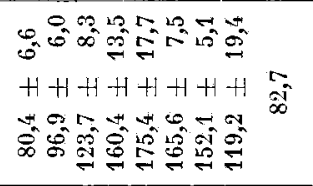 \\
\hline 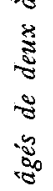 & 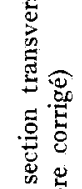 & 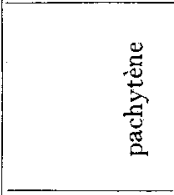 & 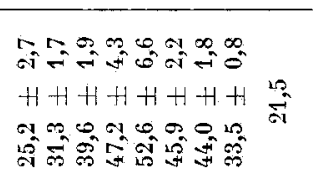 \\
\hline 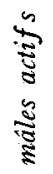 & 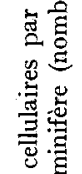 & 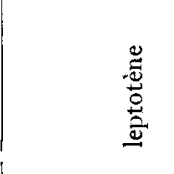 & 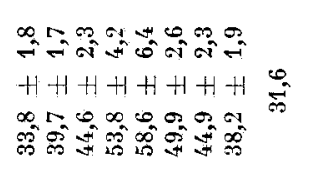 \\
\hline 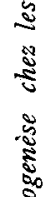 & 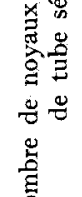 & 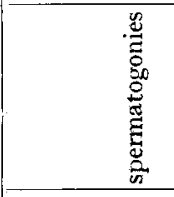 & 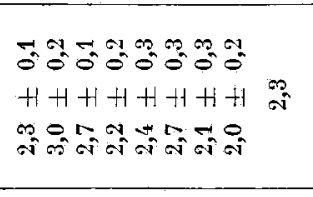 \\
\hline 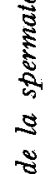 & z & 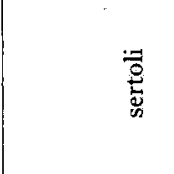 & 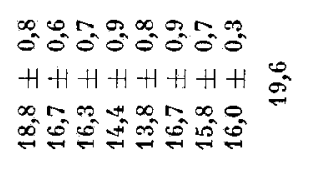 \\
\hline 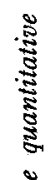 & & 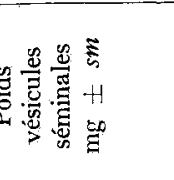 & 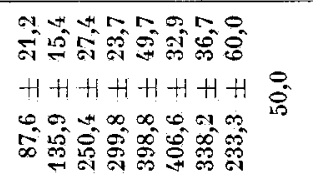 \\
\hline 茎 & & 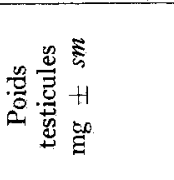 & 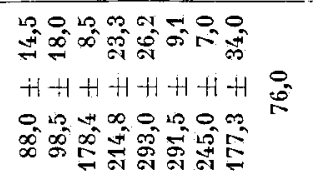 \\
\hline & & 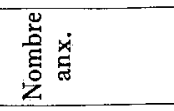 & 융요응ㅇㅇㅇ유유규 \\
\hline & & 产 & 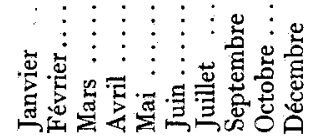 \\
\hline
\end{tabular}


Le nombre d'ovulations augmente avec la taille des femelles (fig. 9).

En effet, on trouve 5,7 $\pm 0,6$ corps jaunes chez les femelles mesurant entre 70 et $80 \mathrm{~mm}, 7, \mathrm{I} \pm 0,4$ corps jaunes, chez celles mesurant entre Iro et I20 mm. Cette différence est significative à $0, \mathrm{I}$ p. Ioo.

Cette augmentation du nombre des corps jaunes n'est peut-être due qu'à un autre facteur, le numéro de la portée.

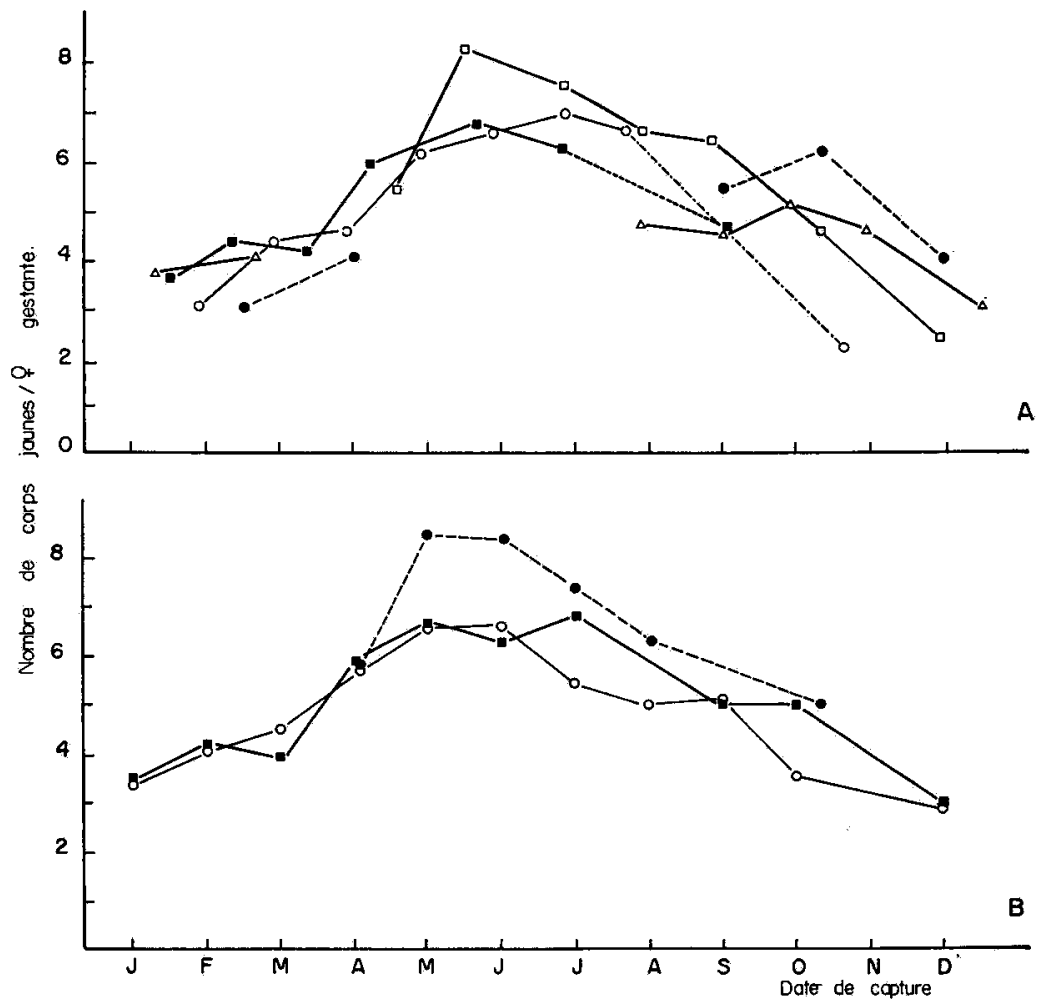

FIG. 8. - Variation du nombre d'ovulations au cours du cycle annuel

A - en fonction de l'année

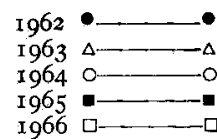

B - en fonction du milieu

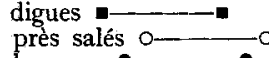

luzernes

Le nombre d'ovulations varie avec le numéro de la portée.

Il est impossible d'après les corps jaunes et les cicatrices placentaires de connaître le numéro de la gestation chez une femelle multipare âgée. Les corps jaunes régressent très rapidement après la mise bas et les cicatrices placentaires ne subsistent pas après une nouvelle gestation. Cependant, chez les femelles très jeunes, de 40 à 60 jours, on peut séparer les femelles primipares et multipares $\left(2^{\mathrm{e}}\right.$ gestation); la deuxième 
mise bas ne pouvant avoir lieu avant 60 jours ( 2 I jours pour la puberté +2 fois 2 I jours de gestation).

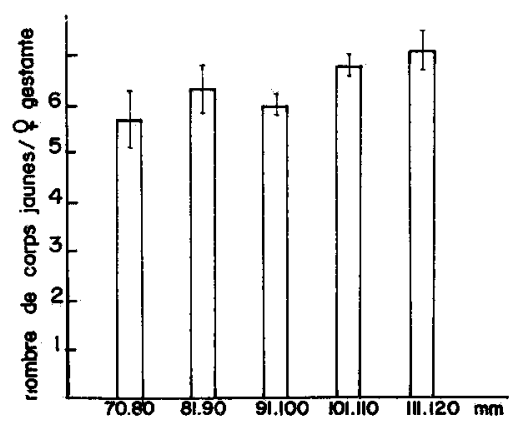

FIG. 9. - Variation du nombre d'ovulations en fonction de la taille des femelles

Chez les femelles de I à 2 mois, le nombre d'ovulations est de $5,9 \pm 0,3$ pour les primipares et de $6,9 \pm 0,3$ pour les multipares (significatif à $0,5 \mathrm{p}$. IOO). Pour ce calcul on a pris en considération uniquement des femelles piégées en avril, mai, juin, pour éviter la variation due à la saison.

Le nombre d'ovulations varie avec le milieu végétal (fig. $8 \mathrm{~B}$ ).

Il n'existe pas de différence entre le nombre de corps jaunes par femelle gestante piégée sur les digues ou sur les prés salés. Par contre, en mai-juin, le nombre d'ovulations des femelles provenant des luzernes est significativement plus élevé que celui des femelles des digues ou des prés salés.
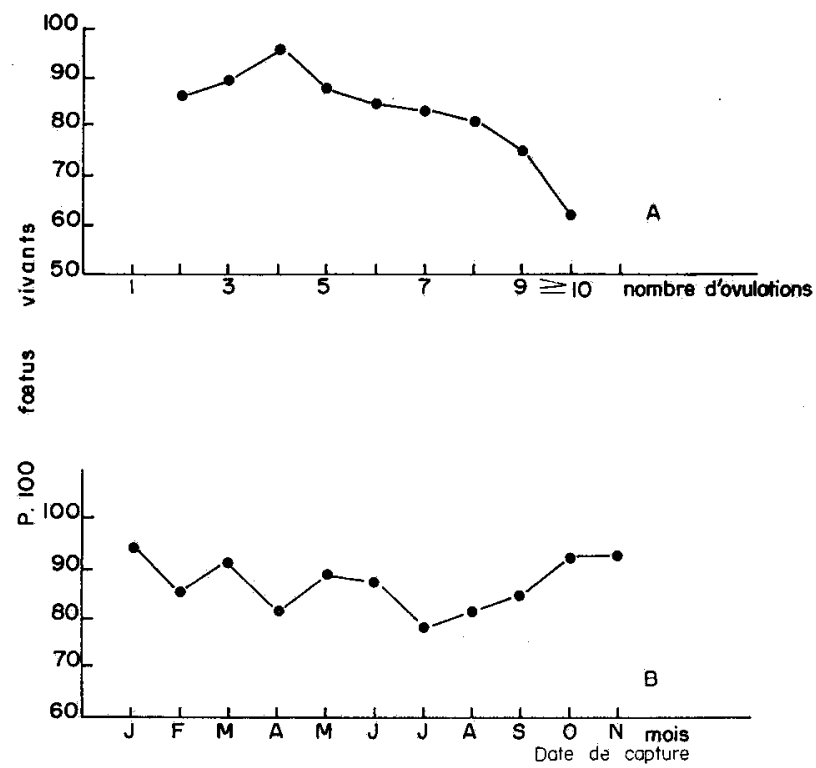

FIG. 10. - Mortalité embryonnaire

A) en fonction du nombre d'ovulations

B) en fonction de la saison 


\section{Mortalité embryonnaire.}

Elle a été estimée d'après les implantations en calculant le pourcentage de fotus vivants par rapport au nombre de corps jaunes.

La mortalité embryonnaire semble augmenter avec la taille des portées (fig. Io A). Pour 4 ovulations, elle est de $4 \mathrm{p}$. Ioo, pour 9 ou Io ovulations elle est de $25 \mathrm{p}$. I0o à 39 p. Ioo.

Par contre, il ne semble pas y avoir de relation nette entre la saison et la mortalité embryonnaire. Cependant, c'est en juillet que la mortalité embyronnaire est la plus importante. En I964, les captures ont été faites après deux semaines de fortes chaleurs ; 25 p. roo de mortalité embryonnaire ont été observés. Ceci est en accord avec les résultats obtenus chez d'autres espèces (Souris - Brebis) concernant l'effet de la température sur la mortalité embryonnaire (fig. Io B).

\section{DISCUSSION}

De l'ensemble des données recueillies au cours des cinq années, on peut aboutir à deux conclusions :

I. Au cours du cycle annuel apparaissent deux catégories d'animaux. Les premiers naissent de février à juin ; leur croissance corporelle et leur maturation sexuelle est rapide, leur durée de vie est courte ; ils meurent entre deux et quatre mois.

Les autres naissent à partir de juin, leur croissance est très lente ; ils n'atteignent pas la maturité sexuelle avant le mois de février suivant. Ce sont eux qui donnent les premières portées de février à mars.

C'est l'existence d'un critère d'âge précis, le poids du cristallin, qui nous a permis de démontrer ce qui n'était jusque-là qu'une hypothèse formulée par de nombreux auteurs. BARBEHEM (I955), BERNARD (I964), avaient remarqué que les animaux nés après juin n'atteignaient pas la maturité sexuelle avant le printemps suivant. Pour HAMILTON (I937), BERNARD (I964), les individus les plus lourds, c'est-à-dire pour eux, les plus âgés, meurent avant la fin de l'hiver. Seuls survivraient les individus nés à la fin de l'été.

SchWARTZ et al. (1964) ont montré 1a succession de deux générations différentes chez les petits rongeurs sauvages, en suivant la vitesse de croissance et de maturation sexuelle dans une population de Microtus coconomus et de Lagurus et lagurus, élevés au laboratoire.

Il ne semble pas exister de régression testiculaire; les animaux capturés de novembre à janvier, qui étaient âgés d'au moins six mois, par conséquent nés avant juillet, étaient en activité sexuelle. Cependant, Huminski (I958), LECYK (I958), reconnaitraient des testicules régressés d'après l'épaisseur et l'aspect fripé de la membrana propria du tube séminifère et d'après une augmentation de volume relatif du tissu interstitiel.

2. Les observations faites pendant cette étude sont en accord avec les travaux d'autres auteurs : la durée de la saison sexelle varie d'une année sur l'autre et suivant le biotope. Le plus souvent, la reproduction s'arrête à partir d'octobre à cause de la 
disparition des individus âgés actifs sexuellement, mais il existe des arrêts précoces dès le mois de juillet, et au contraire, certaines années, on trouve des animaux en reproduction en novembre-décembre.

Il semblerait donc que le photopériodisme n'intervienne pas, ou que partiellement ; ceci est en accord avec les résultats obtenus au laboratoire (MARTINET, I963) où l'apparition de la maturité sexuelle a lieu quelle que soit la durée de la photopériode.

Par contre, on observe une modification de la fertilité au cours de l'année; le nombre de spermatozoïdes produits et le nombre d'ovulations sont supérieurs en jours longs, ce qui est aussi en accord avec les résultats obtenus au laboratoire (MARTINeT, I966 $b$; C. Thibaulit, L. Martinet et $a l$. ., I966).

Il semblerait donc que la lumière intervienne, non sur le nombre d'individus en reproduction, mais sur leur fertilité. Il faudrait chercher l'explication des débuts et des arrêts de saison sexuelle dans d'autres facteurs tels que la présence de végétaux verts, par exemple.

Reçu pour publication en mai 1967 .

\section{REMERCIEMENTS}

Je remercie M. GIBAN, Directeur du Laboratoire des petits Vertébrés, qui a mis à ma disposition son laboratoire de Vendée et M. SprTz qui m'a conseillée au cours de cette étude.

\section{SUMMARY}

SEASONAL REPRODUCTION OF THE FIELD VOLE (" MICROTUS ARVALIS ")

Field Voles were trapped at one month intervals during five years. The age of the animals was estimated by lens weight, and their growth rate, sexual maturity and fertility were investigated.

Growth and sexual maturation rates were found different according to the date of birth. This led us distinguish two successive populations. One is composed of the animals born between March and June : they grow faster, are sexually mature earlier and have a shorter life span. The other one is composed of the animals born in July and later on : they grow slower ; their sexual maturity is delayed until the next spring, and they have a longer life span. The latter is the population who lives the winter out and gives birth to the first litters of the year.

The seasonal reproductive cycle already noticed in the literature was observed. Though the duration of the sexual season is not the same every year, fertility estimated in the male from the importance of epididymous sperm storage and in the female from the number of ovulations and fœtues, always reaches a maximum in April or May.

\section{RÉFÉRENCES BIBLIOGRAPHIQUES}

Amman R. P., 1962. Reproductive capacity of dairy bulls. VIII. Direct and indirect measurement of testicular sperm production. J. Dairy Sci., 45, 774-87I.

BAKER J., RANSON M.,I932. Factors affecting the breeding of the field mouse Microtus agrestis. Light. Proc. roy. Soc. Biol., 110. 313-322.

BARBehem K. R., I955. A field study of growth in Microtus pennsylvanicus. J. Mammal., 36, 533-543. 
Beer J. R., Mac Leod C. F., I96r. Seasonal reproduction in the meadow vole. J. Mammal., 42, 483-489. BernaRD, I964. Note préliminaire sur la reproduction chez le Campagnol des champs Microtusarialis dans le sud de la Belgique. Bull. Inst. agron. Sta. Recher. Gembloux, 32, 275-289.

Chappelier A., I 937 . Mensurations et rapports chez quelques petits rongeurs. Mammalia, 1,`243-256.

Cowan I., Arsenault M. G., I954. Reproduction and growth in the creeping vole, Microtus oregoni. Can. J. Zool., 32, 198-208.

Delost P., I955. Étude de la biologie sexuelle du Campagnol des champs (Microtus arvalis P.). Arch. Anat. Micro. Morph. Exp., 44, I 50-19o.

Dounce A. L., I943. Further studies on isolated cell nuclei of normal rat liver. J. Biol. Chem., 151, 22 I-233.

Greenvald G. S., r957. Reproduction in a coastal California population of the freld mouse, Microtus Californicus. Univ. Calif. Publ. Zool., 54, 421-446.

Hamilton W. J., Jr, I937. Growth and life span of the field mouse. Am. Nat., 71, 500-507.

Huminski S., I958. The automnal involution of the male sexual apparatus in the field vole (Microtus arvalis) Zool. Poloniae. 9, 197-214.

LECYK M., 1958. The automnal involution of the testes in the field vole (Microlus arvalis pallas) Zool. Poloniae, 9, 216-222.

LORD R. D., 1959. The lens as an indicator of age in cottontail rabbits. J. Wildl. Mgmt., 23, 358-360.

MARTinet L., I 963. Etablissement de la spermatogenèse chez le Campagnol des Champs (Microtus arvalis) en fonction de la durée quotidienne d'éclairement. Ann. Biol. anim. Bioch. Biophys., 3, 342-352.

MaRTinet L., I966a. Détermination de l'âge chez le Campagnol des champs (Microtus arvalis pallas) par la pesée du cristallin. Mammalia, 30, 425-430.

Martinet L., I966 b. Modification de la spermatogenèse chez le Campagnol des champs (Microtus arvalis) en fonction de la durée quotidienne d'éclairement. Ann. Biol. anim. Bioch. Biophys., 6, 30I-313.

Maximov A. A., 1948. Fécondité et dynamique des populations de Microtus arvalis (en russe). Svr. Akad. Nauk S. S. S. R. Ser. Biol., 1, 73-82.

Ortavant R., I958. Le cycle spermatogénétique chez le Bélier. Thèse Fac. Sci. Paris.

Raynaud A., 195I. Reproduction en hiver des Campagnols agrestes dans le département du Tarn. Bull. Soc. Zool. France. 76, 188-200.

Schwartz S. S., et al., I964. Biological peculiarities of seasonal generations of rodents, with spécial reference to the problem of senescence in mammals. Acta. Theriologica, 8, II-43.

SpITz F., Ig63. Observations sur le mécanisme des fluctuations de Microtus arvalis à Saint-Michel-en-l'Herm (Vendée). Ann. Épiphyites, 14, 289-29o.

STEIN G. H. W., r 953 . U̇ber Umweltabhängigkeiten bei der vermehrung der feldmaus (Microtus arvalis). Zool. jb. Syst., 82, $65^{2-547 .}$

Thibault C., et al., r966. Regulation of breeding season and ostrous cycles by light and external stimuli in some mammals. 7. anim. Sci., 25, т19-142. 